Chapter 4

\title{
Photonic Crystal Laser Based Gas Sensor
}

\author{
Marcus Wolff, Henry Bruhns, Johannes Koeth, \\ Wolfgang Zeller and Lars Naehle
}

Additional information is available at the end of the chapter

http://dx.doi.org/10.5772/57147

\section{Introduction}

The development of new radiation source technologies has a major impact on the progression of optical trace gas detection [1]. Especially semiconductor diode lasers have proven to be extraordinarily suitable devices for spectroscopic sensors. Their small size and their low acquisition cost are here valuable properties. However, it is particularly advantageous that their emission can spectrally be tuned simply via their operating temperature and operating current. Furthermore, diode lasers can be directly modulated via their injection current. Therefore, they represent particularly suitable radiation sources for photoacoustic spectroscopy because this technique is based on the absorption of modulated radiation and its transformation into a sound wave. As an offset-free technique it enables extremely high detection sensitivity [2].

Continuous-wave (cw) single-frequency diode lasers, like distributed feedback (DFB) lasers, are particularly suitable for spectroscopy because they avoid any cross-sensitivity and enable very selective gas detection $[3,4]$. DFB devices were originally developed for the telecommunication industry and can conveniently be operated at room temperature. Meanwhile, available emission wavelengths cover the entire near-infrared spectral range $(800 \mathrm{~nm}-3000$ $\mathrm{nm})$. Most recently, DFB lasers operating in the mid-infrared were introduced (>3000 nm) [5]. The alternative concept of interband cascade lasers (ICL) covers almost the complete midinfrared from $3 \mu \mathrm{m}$ to $6 \mu \mathrm{m}$ [6]. This wavelength range is extraordinarily important for trace gas detection since many molecules have their strong fundamental vibrational absorption bands in this region, enabling extremely high detection limits. These devices close the gap to quantum cascade lasers (QCL), which are currently available with single-frequency emission wavelengths starting around $4 \mu \mathrm{m}$ (multimode at $3 \mu \mathrm{m}$ ) [7]. 
However, semiconductor lasers suffer from the considerable weakness that their spectral tuning range is limited to a few nanometers only [4]. As a result of that, the analysis of a gas mixture typically requires the use of a separate laser for each gas component. This makes tunable diode laser spectroscopy expensive and too complex for many applications. A wider spectral tuning range would solve the problem and is, therefore, highly desirable for further practical applications.

This study presents a new photoacoustic gas detection scheme based on a widely tunable coupled-cavity photonic crystal laser operating around $1900 \mathrm{~nm}$. Chapter 2 covers the structure and design of this custom-made room temperature continuous-wave laser followed by measurements of the unique performance characteristics. The new device was designed to enable sensitive detection of water vapor $\left(\mathrm{H}_{2} \mathrm{O}\right)$. Chapter 3 describes the photonic crystal laser based gas sensor and presents photoacoustic measurements of water spectra. The concluding Chapter 4 summarizes the results.

\section{Photonic crystal laser}

\subsection{Basics}

\subsubsection{Photonic crystals}

Photonic crystals are materials with a spatial periodicity in their dielectric constant. Under certain conditions, photonic crystals can create a photonic bandgap, i.e. a frequency window in which propagation of electromagnetic radiation through the crystal is inhibited. Light propagation in a photonic crystal is similar to the propagation of electrons and holes in a semiconductor. An electron passing through a semiconductor experiences a periodic potential due to the ordered atomic lattice. The interaction between electron and the periodic potential results in the formation of energy bands and bandgaps. It is not possible for the electron to pass through the crystal if its energy falls in the range of the bandgap. However, defects or a break in the periodicity of the lattice can locally abolish the bandgap and give rise to interesting electronic properties $[7,8]$.

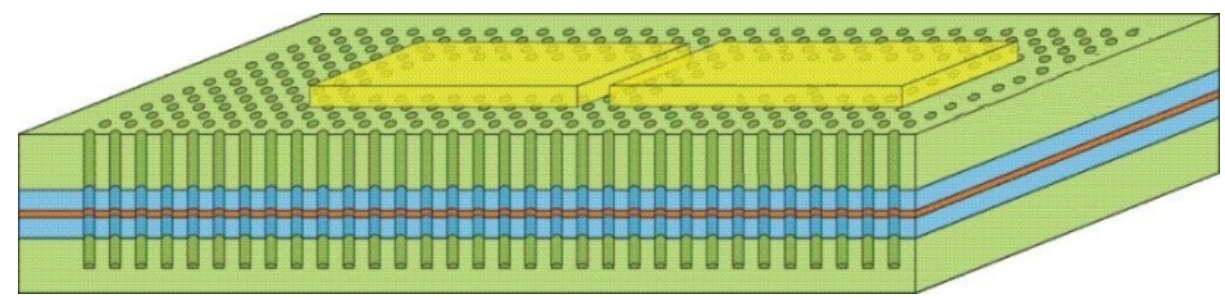

Figure 1. Three-dimensional schematic diagram of photonic crystal structures (courtesy of nanoplus Nanosystems and Technologies $\mathrm{GmbH}$ ). 
Photons in a material with a periodic dielectric constant (index of refraction) experience similar effects. By varying the lattice parameters of these so called photonic crystals, photonic bandgap structures can be obtained that prohibit the propagation of light into the crystal structure. This effect can be used to define optical waveguide structures with very strong lateral light confinement compared to conventional ridge waveguides (of the order of the wavelength) as well as optical filters and wavelength selective reflectors $[9,10]$.

In order for a photon to interact with its periodic environment, its wavelength must be comparable to the periodicity of the lattice. For visible to near-infrared radiation the lattice constant must be in the range of $100 \mathrm{~nm}$ to $1 \mu \mathrm{m}$. Figure 1 depicts a three-dimensional schematic diagram of photonic crystal structures (courtesy of nanoplus Nanosystems and Technologies $\mathrm{GmbH})$. The yellow blocks on top represent contacts.

\subsubsection{Device structure}

The photonic crystal laser we are employing in our optical gas sensor is based on an InAs/ InGaAs quantum dash-in-a-well structure grown on InP substrate by gas source molecular beam epitaxy. A short period superlattice structure is used for photon confinement. Due to their wide spectral gain bandwidth dash-in-a-well structures are particularly well suited for wavelength tuning applications. The photonic crystal is realized by air-semiconductor structures defined by electron beam lithography and etched into the laser structure by an inductively coupled plasma etch step. The structure comprises a hexagonal lattice with a lattice period of $485 \mathrm{~nm}$ and an air fill factor of $30 \%$. The air-filled holes in the semiconductor material exhibit a diameter of $350 \mathrm{~nm}$ and a depth of $3 \mu \mathrm{m}$. These lattice parameters are chosen so that the middle of the photonic bandgap coincides with the device's emission wavelength. The photonic crystal structures are forming multiple lateral and longitudinal photon confinements as well as two independently contacted laser segments. A waveguide is defined by seven rows of missing holes in a ГК oriented photonic crystal. The photonic crystal mirror at the rear end of the laser diode is oriented in ГМ direction in order to present a smooth boundary to the traveling light mode, thus, reducing scattering losses. Coupling of the two laser cavities is provided by a photonic crystal coupling section consisting of two rows of etched holes. The output mirror is formed by a single row of holes. A scanning electron micrograph of the rear, middle and front part of the photonic crystal laser fabricated by nanoplus Nanosystems and Technologies $\mathrm{GmbH}$ in Gerbrunn, Germany can be seen in Figure 2 [11,12].

The photonic crystal laser integrates two longitudinally coupled Fabry-Pérot (FP) resonators. The main advantage of this principle called coupled cavities is that sophisticated grating structures which are difficult and expensive to implement are avoided. Instead, due to the inverse length dependence of mode spacing in a FP resonator, different mode spacings can be realized by implementing different cavity lengths in each segment. Table 1 lists the lengths and according mode spacings of the two photonic crystal laser segments. The coupled cavity laser diode is emitting radiation in the $1.9 \mu \mathrm{m}$ wavelength range. 


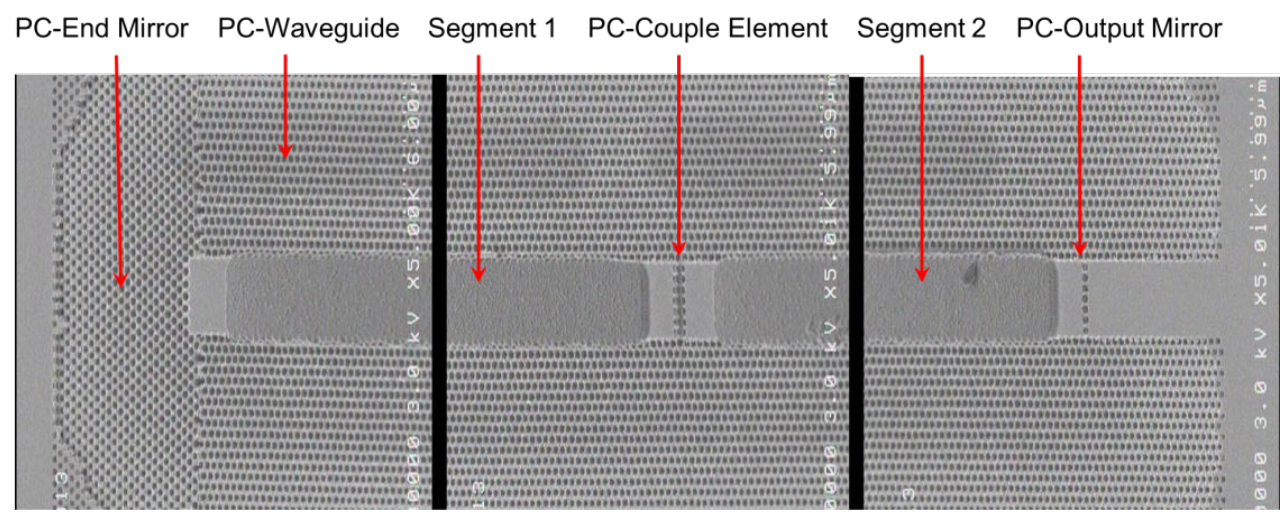

Figure 2. Scanning electron micrograph of the rear, middle and front part of the photonic crystal laser fabricated by nanoplus Nanosystems and Technologies GmbH in Gerbrunn, Germany [11,12].

\begin{tabular}{lcc}
\hline - segment no. & cavity length & mode spacing \\
\hline - 1 (long, rear) & $336 \mu \mathrm{m}$ & $1.59 \mathrm{~nm}$ \\
\hline - 2 (short front) & $241 \mu \mathrm{m}$ & $2.22 \mathrm{~nm}$ \\
\hline
\end{tabular}

Table 1. Cavity lengths and mode spacings of the two photonic crystal laser segments.

\subsection{Characterization}

The optical output power and the spectral emission of the photonic crystal laser were experimentally investigated. The results are presented in the following subsections. However, not all of the laser parameters important for practical applications have been studied yet. Particularly the temporal stability of wavelength and optical power as well as the sensitivity to environmental factors such as ambient temperature and relative humidity have to be examined.

\subsubsection{Emission power}

The photonic crystal laser is working in continuous-wave mode (cw). Both laser segments can be operated separately, each with a maximum current of $120 \mathrm{~mA}$. Similarly to traditional diode lasers, the optical output power of the photonic crystal laser depends on temperature and current. The emission power as function of the operating currents of the two laser segments has been measured using the laser diode controller LDC402B/TED 420 from Profile and a Nova II Laser Power Meter with thermal head 3A-FS-SH from Ophir Optronics.

Figure 3 displays the results recorded at an operating temperature of $20^{\circ} \mathrm{C}$. It is obvious that the power is a relatively complex function of the two currents, compared to a traditional single cavity laser. The power is not proportional to the currents. Therefore, normalization according to the instantaneous optical emission power is mandatory for the exact measurement of absorption spectra. Otherwise falsifications will occur. The threshold currents of the laser 


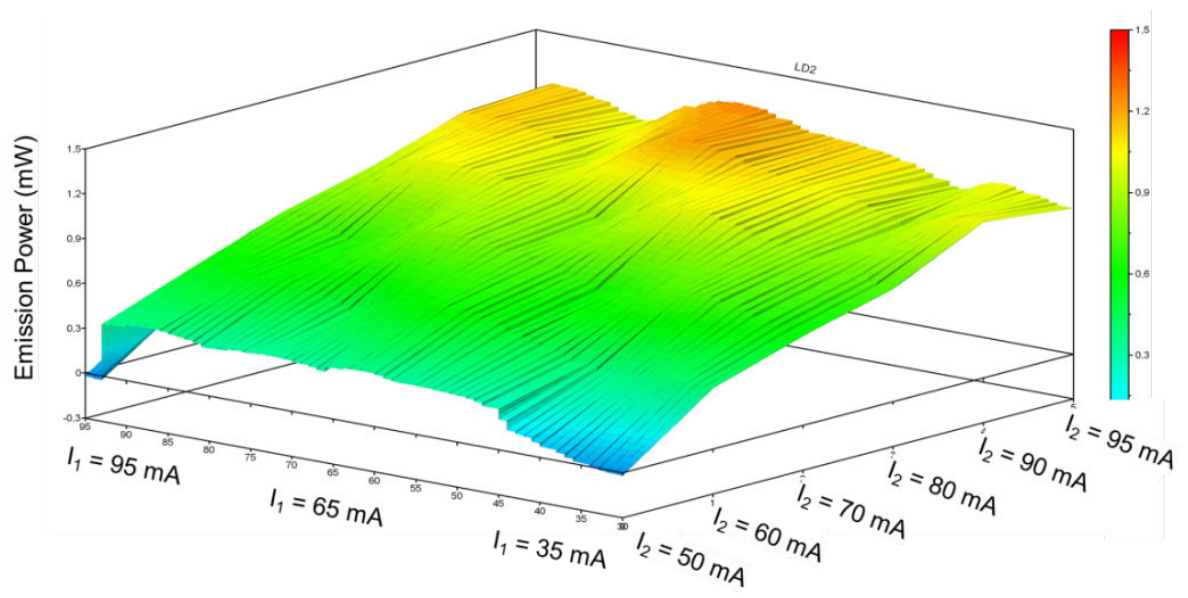

Figure 3. Optical output power of the photonic crystal laser as function of the operating currents of the two laser segments.

segments depend on the operating current of the respective other segment and are between $20 \mathrm{~mA}$ and $35 \mathrm{~mA}$ for segment 1 and between $35 \mathrm{~mA}$ and $50 \mathrm{~mA}$ for segment 2, respectively. The laser provides a maximum emission power of approximately $1.3 \mathrm{~mW}$.

\subsubsection{Emission wavelength}

Similarly to traditional semiconductor lasers, the spectral emission of the photonic crystal device can be tuned by changing the effective index of refraction of the semiconductor material. This can be achieved by varying the temperature or the carrier density. For the purpose of wavelength tuning via temperature, the laser is equipped with a Peltier element (thermoelectric cooler) and a thermo-resistant temperature sensor. Its operating temperature can be adjusted between $10^{\circ} \mathrm{C}$ and $35^{\circ} \mathrm{C}$ (i.e. around room temperature). The carrier density is determined by the operating current.

The two independent laser segments are each supporting several discretely spaced lasing modes (see Table 1). Single mode emission occurs if the overlap between two peaks is sufficiently large, i.e. their wavelengths are approximately equal. Independently changing the two segment's effective indexes of refraction results in a shift of the two mode combs relative to each other, which is accompanied by a significant change in the laser emission wavelength. This is known as the Vernier effect $[13,14]$. The selected segment lengths ensure that a good overlap exists for one pair of laser modes only, for most combinations of electrical currents.

This tuning mechanism for the two coupled cavities of the photonic crystal laser is schematically displayed in Figure 4. The operating current of segment 2, $\mathrm{I}_{2}$, is successively increased while the operating current of segment $1, \mathrm{I}_{1}$, is kept constant. Initially, the "red" mode is emitted because their two counterparts feature the best overlap. Its wavelength can continuously be tuned by current $\mathrm{I}_{2}$ until the accordance between the "green" modes becomes better than that 


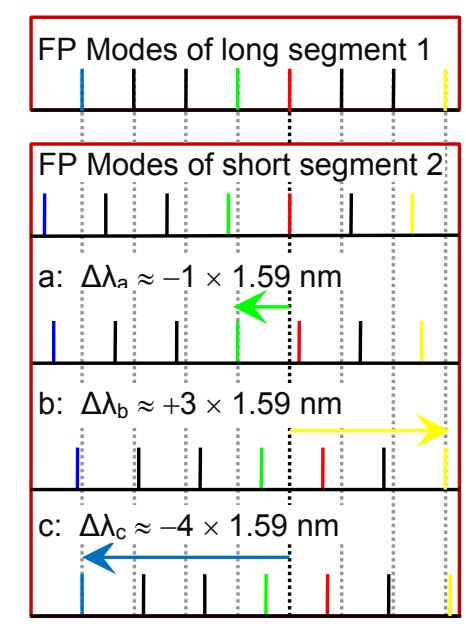

Figure 4. Schematic of the Vernier effect for the two coupled cavities of the photonic crystal laser. $I_{2}$ was increased while $I_{1}$ was kept constant.

of the "red". Consequently, the mode-hop (a) of $\Delta \lambda_{\mathrm{a}}=-1 \times 1.59 \mathrm{~nm}$ takes place. If current $\mathrm{I}_{2}$ is further increased, the hop (b) of $\Delta \lambda_{b}=+3 \times 1.59$ nm to mode "yellow" and, subsequently, hop (c) of $\Delta \lambda_{\mathrm{c}}=-4 \times 1.59 \mathrm{~nm}$ to mode "blue" will occur, each after some continuous tuning. Wavelength tuning with the coupled-cavity photonic crystal laser is, therefore, considered "quasi-continuous" (discretely separated ranges of continuous tuning).

Measurements of the emission wavelength as function of both operating currents were conducted using the laser diode controller LDC402B/TED 420 from Profile and a Fourier transform infrared spectrometer with a DTGS detector (Newport FTIR 80250). Figure 5 shows the emission wavelength as function of $\mathrm{I}_{2}$ while $\mathrm{I}_{1}$ is kept constant at $45 \mathrm{~mA}$. Mode-hops (a), (b), and (c) are marked. A similar behavior can be observed if the current of segment $1, \mathrm{I}_{1}$, is increased while $\mathrm{I}_{2}$ is kept constant. Figure 6 shows the results for $\mathrm{I}_{2}=65 \mathrm{~mA}$. According modehops are marked.

Table 2 lists the complete measuring results recorded at an operating temperature of $15^{\circ} \mathrm{C}$. Wavelengths are in $\mathrm{nm}$. Empty table cells are affiliated with operating parameters which did not allow single-mode operation. During single-mode operation a sidemode-suppressionratio (SMSR) larger than $20 \mathrm{~dB}$ was achieved.

A total tuning range of more than $20 \mathrm{~nm}(1900 \mathrm{~nm}-1920 \mathrm{~nm})$ was observed which is approximately four times as wide as that of traditional semiconductor lasers. Single-mode emission wavelengths in the spectral proximity of water absorption lines are highlighted in Table 2 (1905.7 nm, $1906.7 \mathrm{~nm}, 1907.0 \mathrm{~nm}, 1909.0 \mathrm{~nm}, 1910.6 \mathrm{~nm}, 1913.4 \mathrm{~nm}, 1914.9 \mathrm{~nm})$. The according water vapor absorption spectrum is displayed in Figure 7. It was calculated for a concentration of $1 \%$ at room temperature $\left(20^{\circ} \mathrm{C}\right)$ and atmospheric pressure $(1013 \mathrm{hPa})$ using the HITRAN database [15]. Absorption lines that are spectrally accessible with the photonic crystal laser are marked. The colors are corresponding to the highlighted cells in Table 2. 


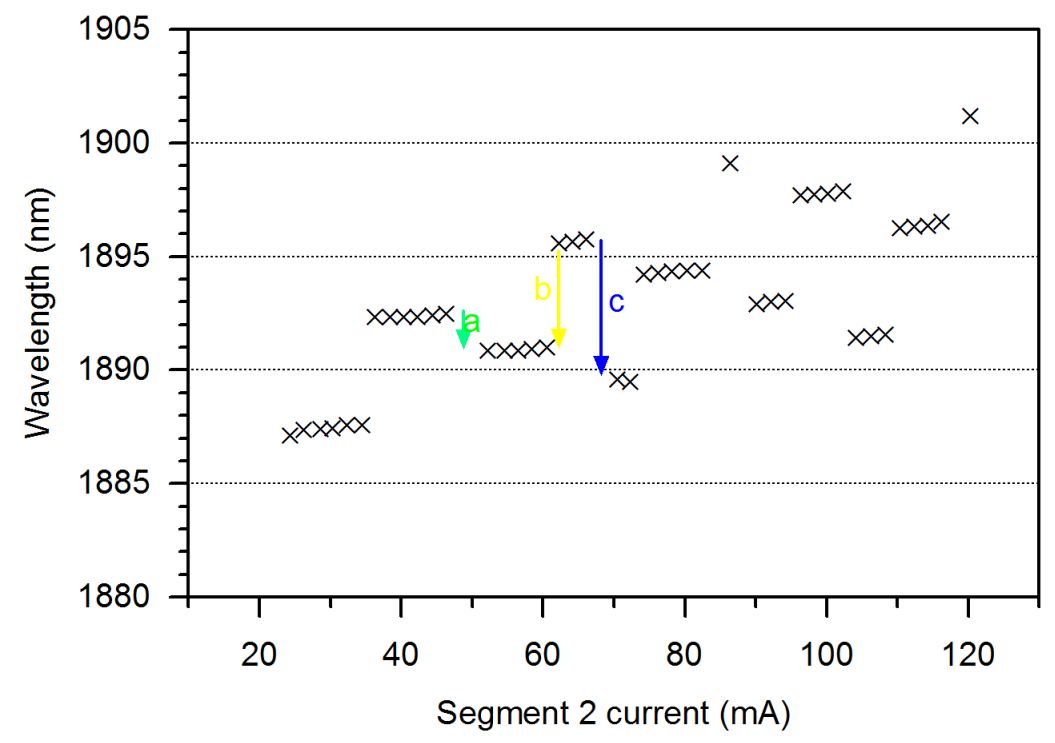

Figure 5. Emission wavelength of the photonic crystal laser as function of segment 2 current, $I_{2},\left(I_{1}=45 \mathrm{~mA}=\right.$ constant).

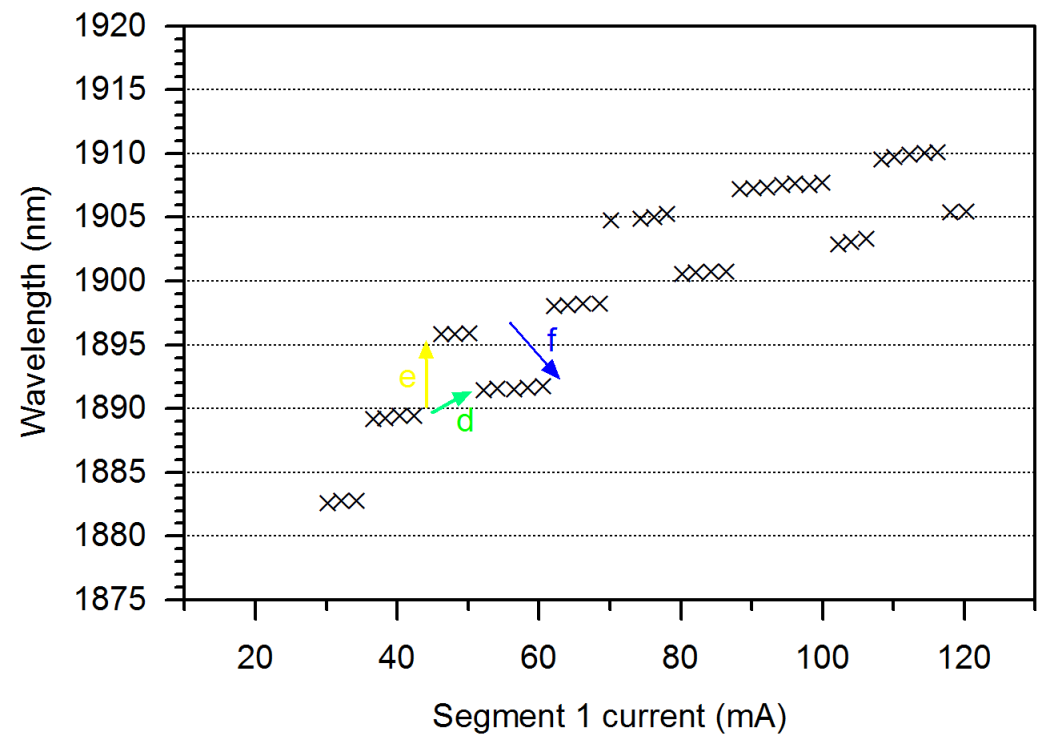

Figure 6. Emission wavelength of the photonic crystal laser as function of segment 1 current, $I_{1},\left(I_{2}=65 \mathrm{~mA}=\right.$ constant). 


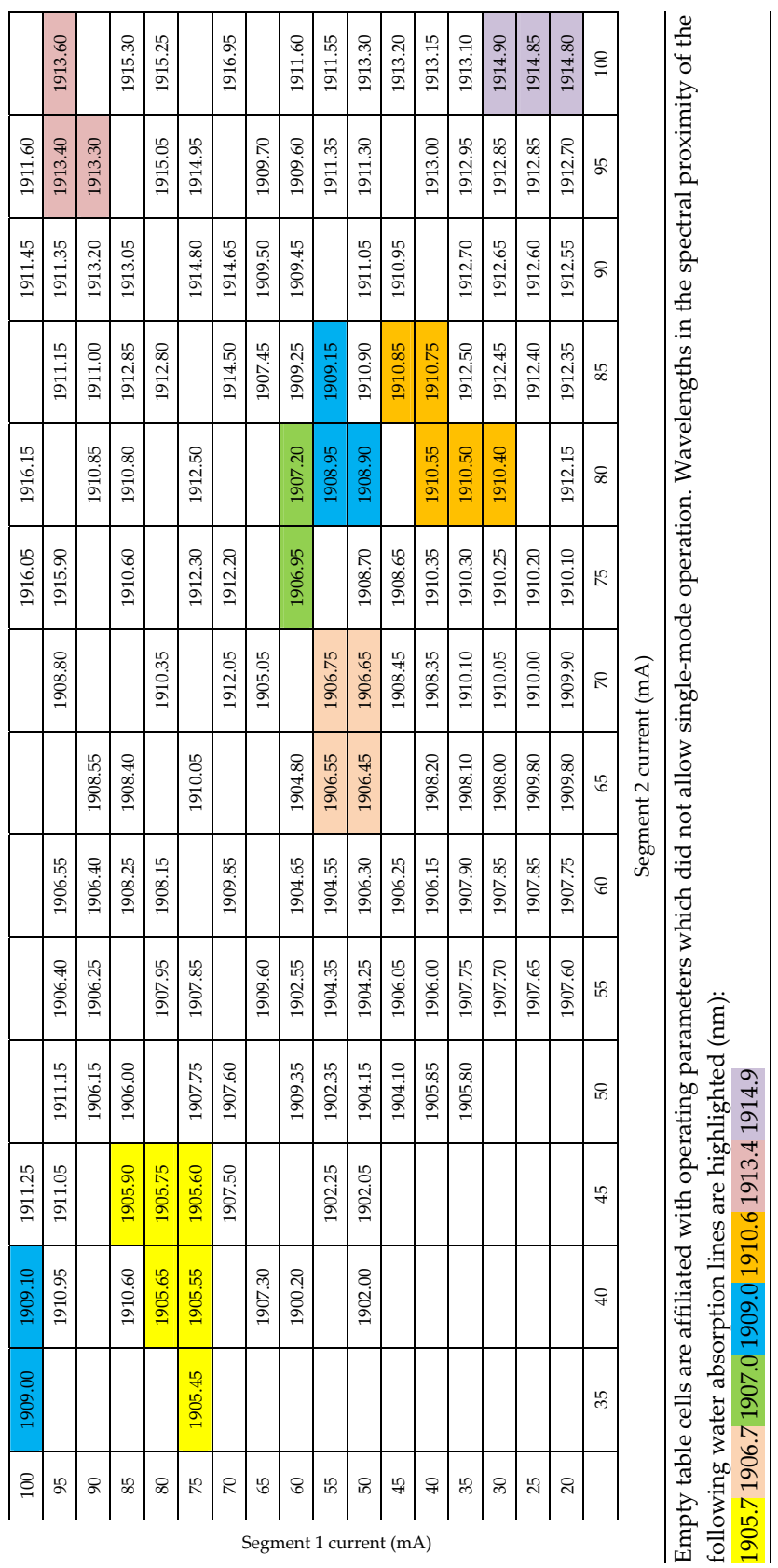

Table 2. Emission wavelength in $\mathrm{nm}$ as function of the operating currents of the two laser segments recorded at an operating temperature of $15^{\circ} \mathrm{C}[16]$. 
Varying the currents in both segments simultaneously enables fine-tuning of the emission wavelength. A combination of coarse and fine tuning mechanisms enables scanning of larger absorption spectra with a single laser device.

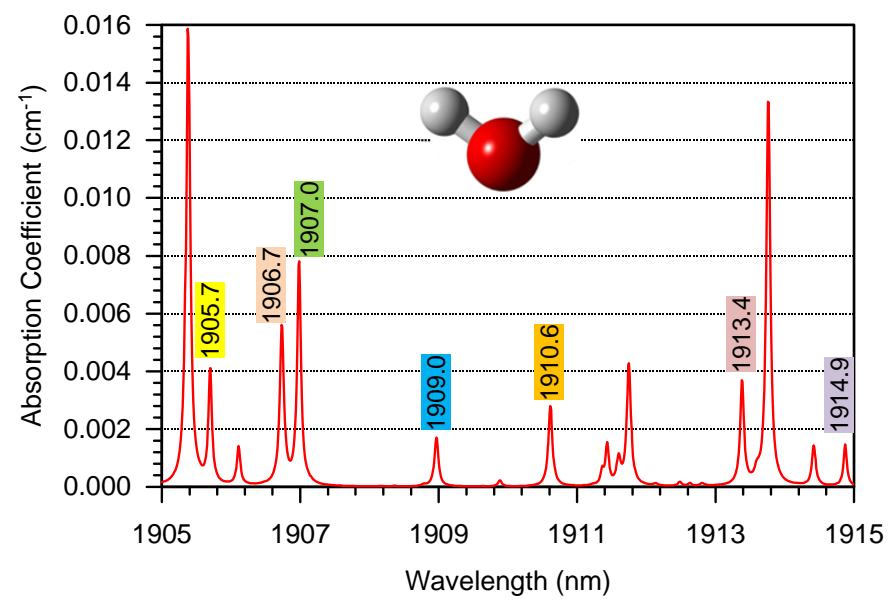

Figure 7. Water vapor absorption spectrum for a concentration of $1 \%$ at room temperature $\left(20^{\circ} \mathrm{C}\right)$ and atmospheric pressure (1013 $\mathrm{hPa})$ according to the HITRAN database [15]. Absorption lines that are spectrally accessible with the photonic crystal laser are marked.

\section{Photoacoustic sensor}

\subsection{Photoacoustic spectroscopy}

The new optical analyzer based on the photonic crystal laser is using photoacoustic spectroscopy. Photoacoustic or optoacoustic spectroscopy (PAS / OAS) is based on the photoacoustic effect that was discovered in 1880 by Alexander Graham Bell [17]. One year later, Wilhelm Conrad Roentgen published a paper on the application of photoacoustic spectroscopy on gas [18].

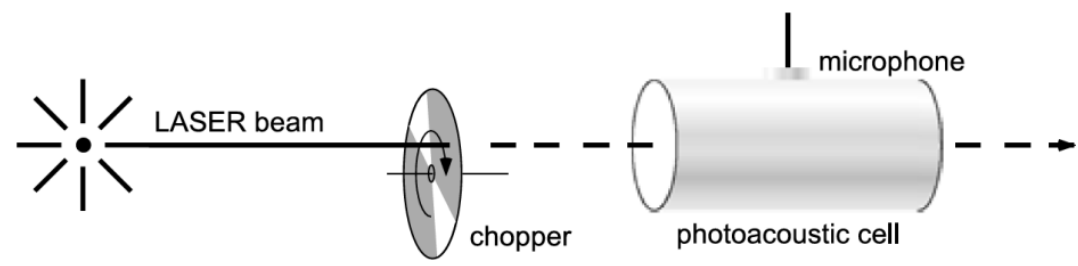

Figure 8. Schematic setup for photoacoustic spectroscopy. 
Just like all other spectroscopic techniques photoacoustics is based on the interaction between matter and electromagnetic radiation [1]. If a sample is irradiated with resonant electromagnetic radiation, i.e. the light frequency coincides with vibrational or rotational eigenmodes of the irradiated molecules, photons are absorbed and a fraction of the molecules is excited into an energetically elevated state.

After the so-called lifetime of the excited state the molecules relax, radiantly or non-radiantly, back into their ground state. PAS is using the part of the energy that is transferred into kinetic energy of collision partners via inelastic collisions (non-radiant relaxation). The velocity of the colliding gas molecules increases. However, an increase of the molecule's average velocity is equivalent with an increase of the temperature of the gas. As long as the sample can be approximately considered an ideal gas, a higher temperature results in a thermo-elastic expansion of the sample which is, at constant volume, equivalent to a local increase of the pressure.

If the irradiation of the sample is interrupted, heat conduction and shear stress lead to a quick deduction of the thermal energy via cell walls and gas volume and, therefore, to a reduction of the pressure back to the initial value. Modulated radiation results in a small periodic pressure variation (typically $10^{-6}$ to $10^{-3} \mathrm{~Pa}$ ) with the modulation frequency of the radiation source, the so-called photoacoustic signal. The detection of this sound wave by a microphone eliminates the offset (usually the atmospheric pressure), since only changes of pressure are detected. The usually following phase-sensitive measurement of the microphone signal with a lock-in amplifier generates a constant signal [2].

The schematic experimental set-up for photoacoustic spectroscopy is shown in Figure 8. It displays a laser whose radiation is mechanically modulated with a chopper and guided through the sample cell that contains the microphone.

Since a photoacoustic signal is produced only when light is absorbed, PAS is, contrary to transmission spectroscopy, considered an offset-free technique. The photoacoustic signal, S, is directly proportional to the concentration of absorbing molecules, Q, the absorption crosssection of the molecular transition, $\sigma$, and the optical output power of the radiation source, $\mathrm{P}_{0}$,

$$
\mathrm{S}=\mathrm{Q} \cdot \sigma \cdot \mathrm{P}_{0} \cdot \mathrm{C}_{\mathrm{CELL}}
$$

The proportionality factor, $\mathrm{C}_{\mathrm{CELL}}$ is often called the cell constant because it primarily depends on the geometry of the sample cell. To a certain extent, however, it is also a function of the beam profile and position, the microphone sensitivity and the modulation function of the light source. The cell constant and, therefore, the sensitivity of a photoacoustic sensor can be considerably improved by taking advantage of acoustical resonances of the sample cell. Modulating the laser radiation at a frequency equivalent to an acoustical mode of the cell enables an enormous enhancement of the signal [19].

Gas sensors based on the photoacoustic effect allow the detection of extremely low concentrations. Detection limits of the order of parts-per-trillion are achievable [2]. Using spectrally fine radiation photoacoustic gas detection is also a very selective method. It is even possible 
to discriminate different isotopes of one molecule [20]. With a spectrally tunable singlefrequency radiation source the absorption coefficient $\alpha$ of the absorbing gas component can directly be measured as function of the wavelength $\alpha(\lambda)$ [21]. We applied this method and recorded water vapor spectra within the continuously tunable wavelength ranges of the photonic crystal laser.

In summary, photoacoustics is an extremely powerful technique and its field of application reaches from gas detection for medical diagnostics [22] to imaging with photoacoustic tomography [23] and is still growing.

\subsection{Experimental setup}

Figure 9 depicts the experimental setup of the photonic crystal laser based gas sensor. Temperature and electrical currents for the two laser segments are controlled using the laser diode controller LDC402B/TED 420 from Profile. The radiation is mechanically modulated by a high-precision chopper (Scitec Instruments; type $300 \mathrm{CD}$ ). After passing the sample cell, the optical power is measured by a thermopile detector (Ophir Laser Measurement Group; type NOVA II with thermal head 3A-FS-SH). Due to the very small absorptions of water vapor in the investigated spectral region the signal qualifies for the power normalization of the photoacoustic signal.

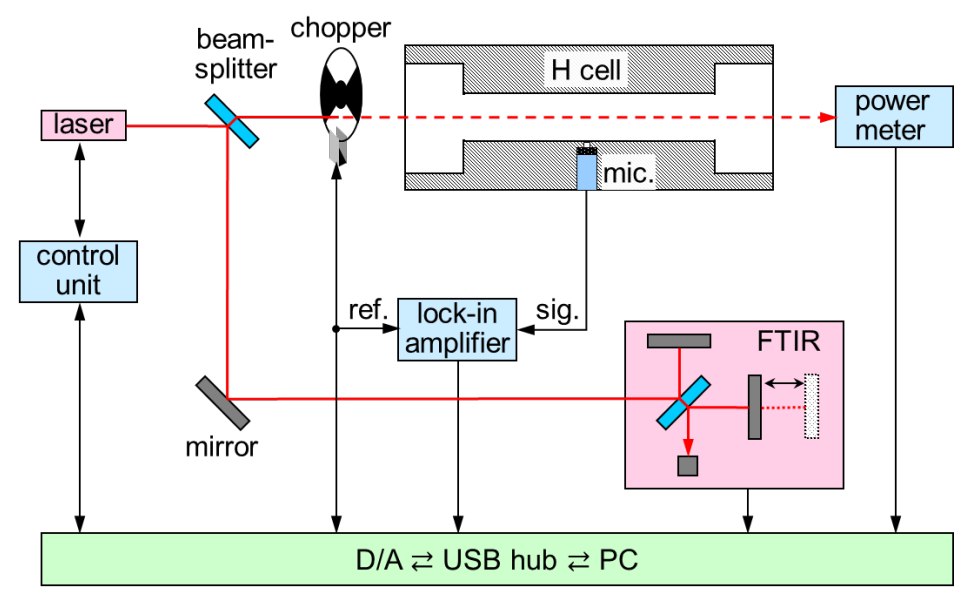

Figure 9. Experimental setup of the photonic crystal laser based gas sensor.

The measuring chamber features the well-established $\mathrm{H}$ geometry [24]. It combines a thin cylindrical resonator of diameter $\mathrm{d}_{1}=6 \mathrm{~mm}$ and length $\mathrm{l}_{1}=60 \mathrm{~mm}$ with two cylindrical buffer volumes of diameter $\mathrm{d}_{2}=24 \mathrm{~mm}$ and length $\mathrm{l}_{2}=30 \mathrm{~mm}$ at the ends. The overall absorption length of the cell is $120 \mathrm{~mm}$. Its first longitudinal mode has a resonance frequency of approximately $2,800 \mathrm{~Hz}$ which was chosen as the modulation frequency. The cell is made of Teflon (PTFE) and the windows are of $\mathrm{CaF}_{2}$. 
The photoacoustic signal is detected by an electret microphone (Primo Microphones GmbH; EN158N), pre-amplified (PAS-Analytik; Amp1) and phase-sensitively recorded (Signal Recovery; Model 7265). The lock-in amplifier uses the output signal of the chopper as reference signal. It measures the absolute value of the photoacoustic signal with a time constant of $1 \mathrm{~s}$. A $\mathrm{CaF}_{2}$ beamsplitter separates a small fraction of the radiation for spectral characterization with a Fourier transform infrared spectrometer (FTIR; MIR ${ }^{\mathrm{TM}} 8025$ Model 80250 manufactured by Newport Corporation).

\subsection{Measurements}

Using the experimental setup described in the previous subsection, photoacoustic spectra of ambient air at atmospheric conditions $\left(1 \% \mathrm{H}_{2} \mathrm{O}, 20^{\circ} \mathrm{C}, 1013 \mathrm{hPa}\right)$ were recorded. At four constant operating currents of laser segment $2\left(\mathrm{I}_{2}=50 \mathrm{~mA} / 55 \mathrm{~mA} / 60 \mathrm{~mA} / 75 \mathrm{~mA}\right)$, the current of laser segment 1 was tuned as far as continuously (mode-hop free) possible. The laser temperature was held constant at $20^{\circ} \mathrm{C}$. Converting current values into corresponding emission wavelengths using previously acquired data (see e.g. Table 2) wavelength dependent absorption plots were generated. The variation in laser output power was compensated by means of normalization according to the instantaneous optical emission power.

Figure 10 displays the four spectra, each one of a single absorption line. As can be seen, the measured photoacoustic signal (crosses) closely tracks the absorption data for water vapor as provided by the HITRAN database [15] (solid line). No cross-sensitivities with other components of air occurred.

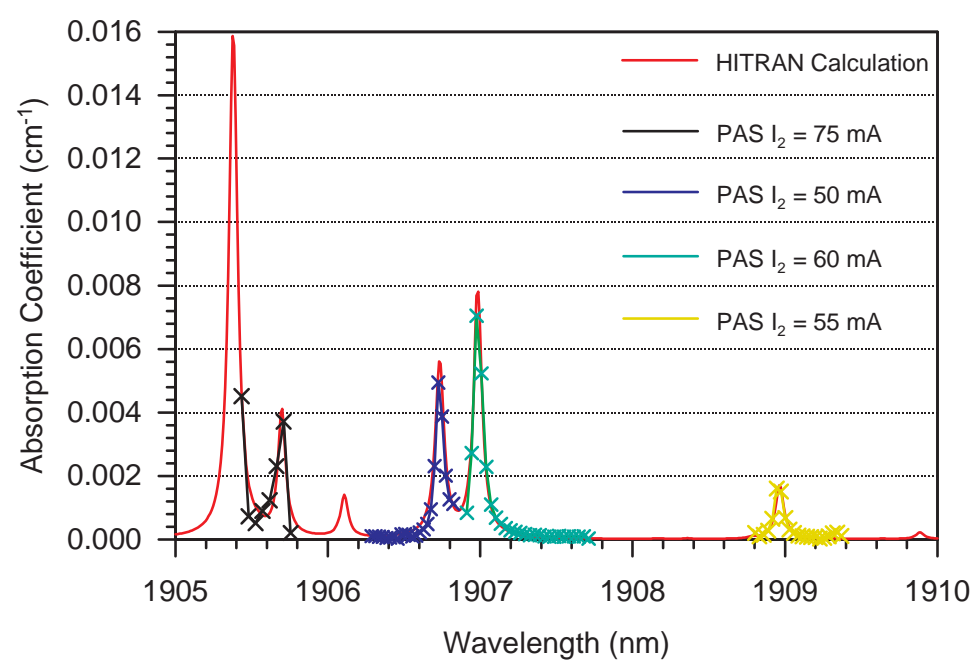

Figure 10. Four photoacoustic spectra of ambient air at atmospheric conditions $\left(1 \% \mathrm{H}_{2} \mathrm{O}, 20^{\circ} \mathrm{C}, 1013 \mathrm{hPa}\right)$, recorded at constant operating current of laser segment $2\left(\mathrm{I}_{2}\right)$ by tuning the operating current of segment $1\left(\mathrm{I}_{1}\right)$ together with a water vapor absorption spectrum according to the HITRAN database [15]. 


\section{Conclusion}

We presented a new optical gas detection scheme based on a photonic crystal laser. We showed emission and operation parameters of this radiation source as well as measured spectra of $\mathrm{H}_{2} \mathrm{O}$ at $1910 \mathrm{~nm}$.

The optical gas sensor based on the photonic crystal laser provides some considerable advantages over those based on traditional semiconductor lasers. The wide spectral tunability of more than $20 \mathrm{~nm}$ allows multigas detection using only one laser. Since the emission wavelength is primarily determined by the two laser currents, its spectral tuning is very fast. Therefore, the analyzer provides a very short response time. Furthermore, the photonic crystal high-reflectance end mirror enables high emission power, which results in combination with photoacoustic spectroscopy in high detection sensitivity. Since these devices do not require the integration of a wavelength selective grating for single-mode emission, it is expected that they will become considerably cheaper than traditional DFB lasers [25,26]. Next generation photonic crystal lasers will feature an even larger tuning range and a higher optical output power of approximately $20 \mathrm{~mW}$. This will qualify these devices even better for a quick and highly sensitive multigas detection using a single laser source.

\section{Author details}

Marcus Wolff1 ${ }^{1 *}$, Henry Bruhns ${ }^{1}$, Johannes Koeth², Wolfgang Zeller ${ }^{2}$ and Lars Naehle ${ }^{2}$

*Address all correspondence to: marcus.wolff@haw-hamburg.de

1 Hamburg University of Applied Sciences, School of Engineering and Computer Science, Department of Mechanical Engineering and Production, Heinrich Blasius Institute for Physical Technologies, Hamburg, Germany

2 Nanoplus Nanosystems and Technologies GmbH, Gerbrunn, Germany

\section{References}

[1] Demtroeder W. Laser spectroscopy. 4th ed. Berlin: Springer; 2009.

[2] Michaelian KH. Photoacoustic Infrared Spectroscopy. 2nd ed. Hoboken (NJ): WileyInterscience; 2011.

[3] Fehér M, Jiang Y, Maier JP. Optoacoustic trace-gas monitoring with near-infrared diode lasers. Applied Optics 1994;33(9):1655-1658. 
[4] Wolff M, Harde H. Photoacoustic Spectrometer based on a DFB-Diode Laser. Infrared Physics and Technology 2000;41(5):283-286.

[5] Naehle L, Belahsene S, von Edlinger M, Fischer M, Boissier G, Grech P, Narcy G, Vicet A, Rouillard Y, Koeth J, Worschech L. Continuous-wave operation of type-I quantum well DFB laser diodes emitting in $3.4 \mu \mathrm{m}$ wavelength range around room temperature. Electronics Letters 2011;47(1):46-47.

[6] Naehle L, Hildebrandt L, Kamp M, Hoefling S. Interband Cascade Lasers: ICLs open opportunities for mid-IR sensing. Laser Focus Word 2013;49(5):70-73.

[7] 2012 Buyer's Guide. Laser Focus Word 2012;48(3):45-46, 61, 64.

[8] Joannopoulos JD. Photonic Crystals: Molding the Flow of Light. 2nd ed. Princeton (NJ): University Press; 2008.

[9] Skorobogatiy M, Yang J. Fundamentals of Photonic Crystal Guiding. Cambridge (MA): University Press; 2008.

[10] Mahnkopf S, Maerz R, Kamp M, Duan GH, Lelarge F, Forchel A. Tunable photonic crystal coupled-cavity laser. IEEE Journal of Quantum Electronics 2004;40:1306-1314.

[11] Mueller M, Scherer H, Lehnhardt T, Roessner K, Huemmer M, Werner R, Forchel A. Widely tunable coupled cavity lasers at $1.9 \mu \mathrm{m}$ on GaSb. IEEE Photonics Technology Letters 2007;19:592-594.

[12] Naehle L, Zimmermann C, Zeller W, Bruckner K, Sieber H, Koeth J, Hein S, Hofling S, Forchel A. Widely tunable photonic crystal coupled cavity laser diodes based on quantum-dash active material. Proceedings of the 20th International Conference on Indium Phosphide \& Related Materials, IPRM 09, 10-14 May 2009; Newport Beach, CA. Red Hook (NY): Curran Associates; 2009. pp.28-30. http://dx.doi.org/10.1109/ ICIPRM.2009.5012418

[13] Zeller W, Legge M, Seufert J, Werner R, Fischer M, Koeth J. Widely tunable laterally coupled distributed feedback laser diodes for multispecies gas analysis based on InAs/InGaAs quantum-dash material. Applied Optics 2009;48:B51-B56.

[14] Bauer A, Mueller M, Lehnhardt T, Roessner K, Huemmer M, Hofmann H, Kamp M, Hoefling S, Forchel A. Discretely tunable single-mode lasers on GaSb using two-dimensional photonic crystal intracavity mirrors. Nanotechnology 2008;19:235202.

[15] Heinrich J, Langhans R, Seufert J, Hofling S, Forchel A. Quantum cascade microlasers with two-dimensional photonic crystal reflectors. IEEE Photonics Technology Letters 2007;19(23):1937-1939.

[16] Rothman LS, et al. The HITRAN2012 molecular spectroscopic database. Journal of Quantitative Spectroscopy and Radiative Transfer 2013 (in press). http://dx.doi.org/ 10.1016/j.jqsrt.2013.07.002i 
[17] Datasheet 1900nm PK-Laser 362-6-18. nanoplus Nanosystems and Technologies $\mathrm{GmbH} ; 2008$.

[18] Bell AG. On the Production and Reproduction of Sound by Light. American Journal of Science 1880;20:305-309.

[19] Roentgen WC. Versuche ueber die Absorption von Strahlen durch Gase; nach einer neuen Methode ausgeführt. Bericht der Oberhessischen Gesellschaft für Natur- und Heilkunde 1881;XX:52-58.

[20] Wolff M, Baumann B, Kost B. Shape-optimized photoacoustic cell: Experimental confirmation and numerical consolidation. Int. J. Thermophysics 2012;33(10):1953-1959.

[21] Wolff M, Harde H, Groninga H. Isotope-Selective Sensor based on PAS for Medical Diagnostics. Journal de Physique IV 2005;125:773-775.

[22] Wolff M, Rhein S, Bruhns H, Nähle L, Fischer M, Koeth J. Photoacoustic Methane Detection using a novel DFB-type Diode Laser at $3.3 \mu \mathrm{m}$. Sensors and Actuators B: Chemical 2013;187:574-577.

[23] Germer M, Wolff M, Harde H. Photoacoustic NO Detection for Asthma Diagnostics. Proc. SPIE 2009;7371:73710Q.

[24] Wang LV, Hu S. Optoacoustics/Deep Tissue Imaging: Photoacoustic tomography is ready to revolutionize. Science 2012;335:1458-1462.

[25] Nodov E. Optimization of resonant cell design for optoacoustic gas spectroscopy (Htype). Applied Optics 1978;17:1110-1119.

[26] Wolff M, Gebhardt S. Photonic crystal laser for photoacoustic spectroscopy. Proceedings of the 15th International Conference on Photoacoustic and Photothermal Phenomena, ICPPP15, 19-23 Jul 2009, Leuven, Belgium. Red Hook (NY): Curran Associates; 2009. p.323.

[27] Wolff M, Koeth J, Zeller W, Gebhardt S. Photonic crystal laser based gas detection. Proceedings of the 4th EPS-QEOD Europhoton Conference, 29 Aug - 03 Sep 2010, Hamburg, Germany. Mulhouse, France: European Physical Soc; 2010. p.30. 
